\title{
IN SILICO DOCKING ROSELLE (HIBISCUS SABDARIFFA L.) CALYCES FLAVONOIDS AS ANTIMALARIAL AGAINST PLASMEPSIN 1 AND PLASMEPSIN 2
}

\author{
NERDY* \\ Department of Pharmacy, Academy of Pharmacy Yayasan Tenaga Pembangunan Arjuna, Pintubosi, Laguboti, Toba Samosir, Sumatera \\ Utara, 22381, Indonesia. Email: nerdy190690@gmail.com
}

Received: 07 May 2017, Received and Accepted: 23 June 2017

\section{ABSTRACT}

Objective: Study the in silico plasmepsin 1 and plasmepsin 2 inhibition antimalarial effects of roselle (Hibiscus sabdariffa L.) calyces flavonoids compared to artemisinin as astandard compound for antimalarial to inhibit plasmepsin 1 and plasmepsin 2.

Methods: Partition coefficient was predicted by the ChemDraw Ultra. In silico molecular docking was done by Protein-Ligand ANT System. Visualization was done by Yet Another Scientific Artificial Reality Application. Connector for Windows operation system to Linux operation system was done by Co Pendrive Linux. Three dimensions enzyme structure models used in this research were plasmepsin 1 and plasmepsin 2 with the protein data bank code 3QS1 and 1LEE obtained through the website http://www.rcsb.org/pdb. Two dimensions and three dimensions conformation model of compounds were generated by Marvin Sketch.

Results: Partition coefficient of roselle calyces flavonoids quercetin, gossypetin, hibiscetin, and artemisinin, respectively, were $0.58,-0.44,-0.43$, and 3.17. Higher partition coefficient means easier to penetrate into the cell. Docking score of roselle calyces flavonoids quercetin, gossypetin, hibiscetin, and artemisinin to plasmepsin 1 , respectively, were $-70.1989,-70.9454,-70.5870$, and -61.7685 to plasmepsin 2 , respectively, were $-73.8620,-76,0086,-78.8930$, and -61.7437 . Lower docking score means a better potential activity to protein enzyme.

Conclusion: Roselle calyces flavonoids (quercetin, gossypetin, and hibiscetin) show the stronger activity than artemisinin to inhibit plasmepsin 1 and plasmepsin 2.

Keywords: In Silico, Hibiscus sabdariffa, Flavonoids, Antimalarial, Plasmepsin 1, Plasmepsin 2.

(c) 2017 The Authors. Published by Innovare Academic Sciences Pvt Ltd. This is an open access article under the CC BY license (http://creativecommons. org/licenses/by/4. 0/) DOI: http://dx.doi.org/10.22159/ajpcr.2017.v10i10.19770

\section{INTRODUCTION}

Based on data from the World Health Organization, malaria disease in Indonesia is a disease with the top 10 ranking causing death in children under 5 years old. Patients with malaria have increased the number of patients from 2012 to 2013. There are 212 million malaria cases worldwide in 2015 [1]. Along the Cambodia-Thailand border, Plasmodium falciparum has become resistant to almost all available antimalarial medicines. There is a real risk that multidrug resistance will soon emerge in other parts of the subregion as well [2]. Because of the spread of multidrug resistance malaria disease, it is urgent need for new antimalarial drugs to prevent antimalarial medicines resistance. Research for alternative drugs is becoming mandatory to prevent and cure malaria.

Drugs from the natural materials or synthetic have many benefits. Medicines from natural materials there are efficacious as antidiabetic [3], antimalarial [4], anticancer [4-6], antibacterial [7,8], and antiaging $[9,10]$. Roselle calyces highly rich in vital minerals such as iron, copper, calcium, magnesium, manganese required for healthy growth in humans, and phytochemical such as glycosides $(0.13 \%)$, saponnins (0.96\%), phenols (1.10\%), alkaloids (2.14\%), tannins $(17.00 \%)$, and flavonoids $(20.08 \%)$ can treat many diseases and conditions [11]. Flavonoids can provide a good antimalarial effect [12]. Roselle calyces contains flavonoids, such as: quercetin, gossypetin, and hibiscetin [13]. However, flavonoids from roselle calyces have not been studied as antimalarial. In this study, researcher interested to examine the antimalarial effects of roselle calyces flavonoids by in silico.

Aspartic proteases called plasmepsin are present in different species of Plasmodium falciparum. With the use of in silico structure-based drug design approach, the differences in binding energies of the substrate and inhibitor were exploited between target sites of parasite and human. Stronger interactions are shown by several molecules as compared to the reference molecules which have shown to be the potential as drug candidates [14]. The protein enzyme plasmepsin 1 and plasmepsin 2 belong to the aspartic proteases that coordinate with cysteine proteases in the process of hemoglobin degradation in the parasite's food vacuole and are considered good drug targets. Artemisinin could inhibitplasmepsin 1 and plasmepsin 2 that can act as antimalarial agent [15]. In this research, researcher will compare the antimalarial activity to inhibit plasmepsin 1 and plasmepsin 2 of roselle calyces flavonoids to artemisinin as a standard antimalarial drug. Fig. 1 shows the roselle calyces flavonoids and artemisinin chemical structure.

Computational methods are being developed to predict the druglikeness of compounds. Thus, drug discovery is already on the road toward electronic Research and Development. In silico approaches contribute significantly to early pharmaceutical research and are

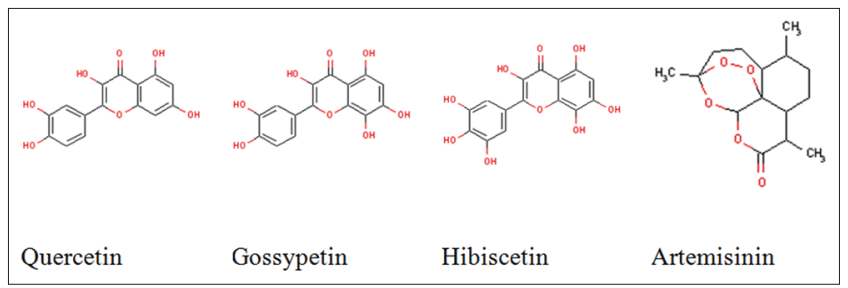

Fig. 1: Roselle calyces flavonoids and artemisinin chemical sstructure 
Table 1: Partition coefficient and docking score of compound with the receptor protein enzyme plasmepsin 1 and plasmepsin 2

\begin{tabular}{|c|c|c|c|c|c|c|}
\hline \multirow[t]{2}{*}{ Number } & \multirow[t]{2}{*}{ Compound } & \multicolumn{2}{|c|}{ Molecular } & \multirow[t]{2}{*}{ Partition coefficient } & \multicolumn{2}{|l|}{ Docking score } \\
\hline & & Formula & Weight & & Plasmepsin 1 & Plasmepsin 2 \\
\hline 1 & Quercetin & $\mathrm{C}_{15} \mathrm{H}_{10} \mathrm{O}_{7}$ & 304.25 & 0.58 & -70.1989 & -73.8620 \\
\hline 2 & Gossypetin & $\mathrm{C}_{15}^{15} \mathrm{H}_{10} \mathrm{O}_{8}$ & 318.24 & -0.44 & -70.9454 & -76.0086 \\
\hline 3 & Hibiscetin & 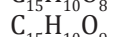 & 334.23 & -0.43 & -70.5870 & -78.8930 \\
\hline 4 & Artemisinin & $\mathrm{C}_{15}^{15} \mathrm{H}_{22} \mathrm{O}_{5}$ & 282.33 & 3.17 & -61.7685 & -61.7437 \\
\hline
\end{tabular}

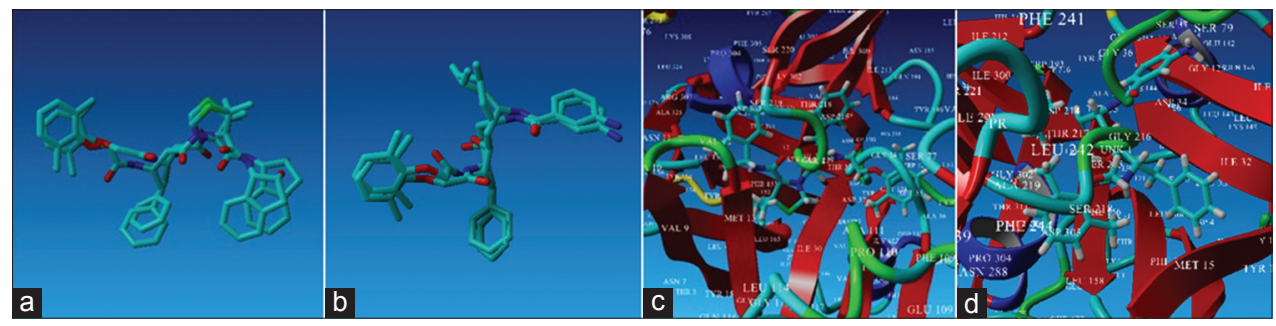

Fig. 2: Overlapping between native ligands after redocking and native ligands before redocking ([a] KNI; [b] R36) and interaction between native ligands after redocking to its original binding pockets and protein enzyme ([c] KNI and plasmepsin 1; [d] R36 and plasmepsin 2)

especially important in target discovery and lead discovery [16]. Therefore, there is an increased interest to identify potential activities of roselle calyces flavonoids to inhibit protein enzyme plasmepsin 1 and plasmepsin 2 as the malaria protein enzyme target compared with artemisinin as the standard compound by in silico docking.

\section{METHODS}

Fujitsu T Series (T4310) operated by Windows 7 Home Premium, Intel ${ }^{\circledR}$ Core $^{T M} 2$ Duo central processing unit T660 ${ }^{\circledR} 2.20 \mathrm{GHz}, 32$-Bit, read only memory $320 \mathrm{~GB}$, and random access memory $4.00 \mathrm{~GB}$ was used to run the molecular docking process. Partition coefficient was predicted by the ChemDraw Ultra program. In silico molecular docking was done by Protein-Ligand ANT System program. Visualization was done by Yet Another Scientific Artificial Reality Application program. Connector for Windows operation system to Linux operation system was done by Co Pendrive Linux program. Three dimensions enzyme structure models used in this research were plasmepsin 1 and plasmepsin 2 with the protein data bank code 3QS1 and 1LEE obtained through the website http://www.rcsb.org/pdb. Two dimensions and three dimensions conformation model of quercetin, gossypetin, hibiscetin, and artemisinin as the standard plasmepsin inhibitors were generated by Marvin Sketch program.

\section{RESULTS AND DISCUSSIONS}

The protein enzyme plasmepsin 1 and plasmepsin 2 with code 3 QS1 and 1LEE have the native ligand KNI and R36. The native ligands were extracted and redocked into its original binding pockets. The root mean square deviation values resulted from these overlapping between native ligands after redocking to its original binding pockets and native ligands before redocking to its original binding pockets were $1.7099 \AA$ and $1.2918 \AA$, which was $<2.0000 \AA$, a value typically used in evaluating the success of docking algorithms, indicating the docking methods was valid [17]. Fig. 2 shows the overlapping between native ligands after redocking and native ligands before redocking and interaction between native ligands after redocking to its original binding pockets and protein enzyme.

Prediction of the partition coefficient value of compound is an initial test to predict the solubility of compounds in the water phase and the oil phase. Testing the antimalarial effects of compounds were done by in silico docking between protein enzyme plasmepsin 1 and plasmepsin 2 with roselle calyces flavonoids (hibiscetin, gossypetin, and quercetin) as the test compound and artemisinin as the standard compound resulting the docking score. Table 1 shows the partition coefficient and

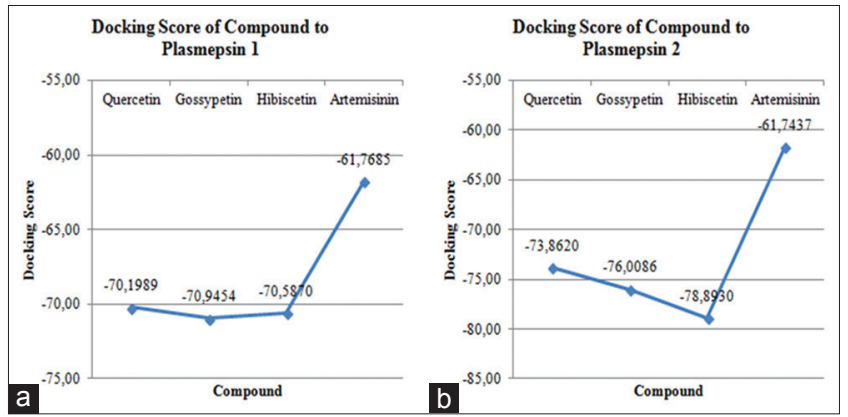

Fig. 3: Relation graph between compound and docking score results protein enzyme (a) plasmepsin 1, (b) plasmepsin 2

the docking score of test compound and standard compound with the receptor protein enzyme plasmepsin 1 and plasmepsin 2. Fig. 3 shows the relation graph between compound and docking score results on protein enzyme.

From the partition coefficient data, it can be seen that artemisinin as a standard compound for antimalarial to inhibit plasmepsin 1 and plasmepsin 2 have a higher partition coefficient than roselle calyces flavonoids as the test compound. Higher partition coefficient of artemisinin as a standard compound for antimalarial to inhibit plasmepsin 1 and plasmepsin 2 than quercetin, gossypetin, and hibiscetin means that the solubility of artemisinin as a standard compound for antimalarial to inhibit plasmepsin 1 and plasmepsin 2 in oil phase was higher than quercetin, gossypetin, and hibiscetin. Higher solubility in oil phase of artemisinin as a standard compound for antimalarial to inhibit plasmepsin 1 and plasmepsin 2 than quercetin, gossypetin, and hibiscetin means the ability to penetrate into the cell of artemisinin as a standard compound for antimalarial to inhibit plasmepsin 1 and plasmepsin 2 was easier than quercetin, gossypetin, and hibiscetin. Partition coefficient only means the ability of the drug to soluble in the oil phase (cell membrane) to penetrate into the cell. The activity of the drug does not only depend on the penetration ability of the drug to penetrate into the cell but also depend on the binding ability of the drug to bind with the binding pocket. The activity of the drug to bind with the binding pocket depends on the structure.

From the docking score data, it can be seen that roselle calyces flavonoids quercetin, gossypetin, and hibiscetin as the test compound 


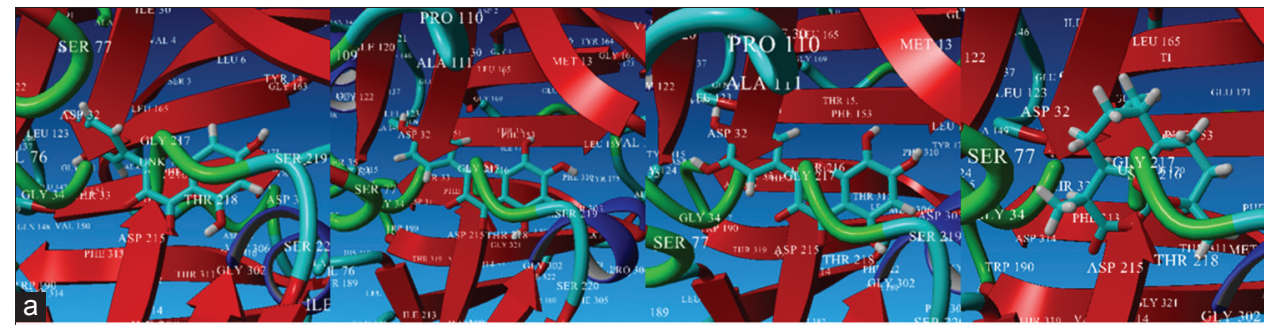

(A2)

(A3)
(A4)

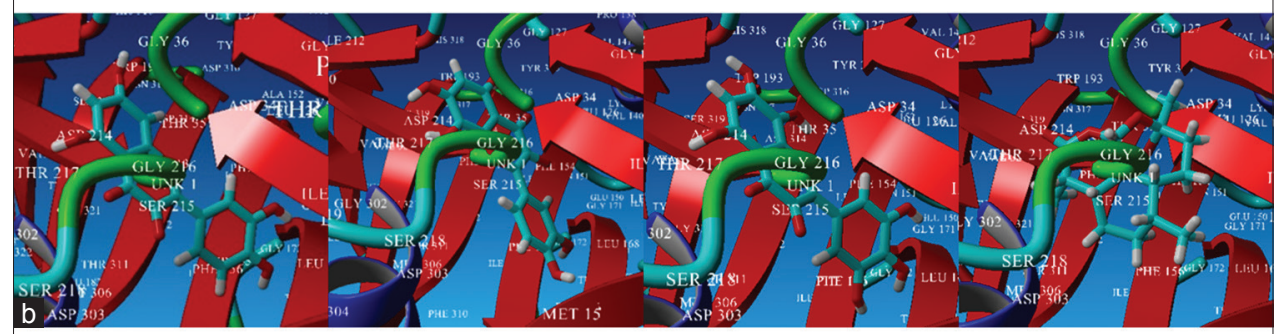

(B1) (B2) (B3) (B4)

Fig. 4: Visualization of interaction between compounds (1: quercetin; 2: gossypetin; 3: hibiscetin; 4: artemisinin) and protein enzyme ([a] plasmepsin 1; [b] plasmepsin 2)

have lower docking score on molecular docking to plasmepsin 1 and plasmepsin 2 than artemisinin as a standard compound for antimalarial to inhibit plasmepsin 1 and plasmepsin 2. Lower docking score on molecular docking to plasmepsin 1 and plasmepsin 2 of quercetin, gossypetin, and hibiscetin than artemisinin as a standard compound for antimalarial to inhibit plasmepsin 1 and plasmepsin 2 means the energy needed to penetrate into protein enzyme binding pocket plasmepsin 1 and plasmepsin 2 of quercetin, gossypetin, and hibiscetin were lower than artemisinin as a standard compound for antimalarial to inhibit plasmepsin 1 and plasmepsin 2. Lower energy need to penetrate into protein enzyme binding pocket plasmepsin 1 and plasmepsin 2 of quercetin, gossypetin, and hibiscetin than artemisinin as a standard compound for antimalarial to inhibit plasmepsin 1 and plasmepsin 2 means the ability to penetrate into the protein enzyme binding pocket plasmepsin 1 and plasmepsin 2 of quercetin, gossypetin, and hibiscetin were easier than artemisinin as a standard compound for antimalarial to inhibit plasmepsin 1 and plasmepsin 2 . Easier ability to penetrate into the protein enzyme binding pocket of quercetin, gossypetin, and hibiscetin than artemisinin as a standard compound for antimalarial to inhibit plasmepsin 1 and plasmepsin 2 means the activity to interact with protein enzyme plasmepsin 1 and plasmepsin 2 of quercetin, gossypetin, and hibiscetin were stronger than artemisinin as a standard compound for antimalarial to inhibit plasmepsin 1 and plasmepsin 2. The docking score represents the binding affinity of the ligand to the enzyme, smaller docking score value shows stronger interaction [18]. Fig. 4 shows the visualization of interaction between compounds and protein enzyme plasmepsin 1 and plasmepsin 2 .

Increasing costs of drug development and reduced number of new chemical entities have been a growing concern for new drug development in recent years. Therefore, there is a need for the use of alternative tools to get answers on the efficacy and safety faster, with more certainty and at lower cost. One such alternative tool is the in silico drug design or the computer-aided drug design. In silico drug design can play a significant role in all stages of drug development from the preclinical discovery stage to late stage clinical development [19]. The results obtained in silico screening have shown that it represents the best way to get accurate results in a very short period and saving manner [20]. Although the application of docking and scoring has led to some remarkable successes, there are still some major challenges ahead [21].

\section{CONCLUSIONS}

Flavonoids are abundantly contained in roselle calyces can inhibit the plasmepsin 1 and plasmepsin 2. All the roselle calyces flavonoids (quercetin, gossypetin, and hibiscetin) show the lower docking score on molecular docking to plasmepsin 1 and plasmepsin 2, lower energy need to penetrate into protein enzyme binding pocket plasmepsin 1 and plasmepsin 2 , easier to penetrate into protein enzyme binding pocket plasmepsin 1 and plasmepsin 2, stronger activity to interact with the protein enzyme plasmepsin 1 and plasmepsin 2 than artemisinin as the standard plasmepsin 1 and plasmepsin 2 inhibition drug.

\section{REFERENCES}

1. World Health Organization; 2016. Available from: http://www.who.int/ malaria/en.

2. World Health Organization; 2017. Available from: http://www.who.int/ malaria/media/artemisinin_resistance_qa/en.

3. Nerdy. In Silico docking of chemical compounds from roselle calyces (Hibiscus Sabdariffa L.) as antidiabetic. Int J Chemtech Res 2015;7(1):148-52.

4. Nerdy. In Silico study of sesquiterpene lactone compounds from South Africa leaves (Vernonia amygdalina Del.) as antimalarial and anticancer. Int J Pharmtech Res 2015;7(1):47-53.

5. Nerdy, Putra ED, Haro G, Harahap U. In silico screening of hesperetin and naringenin ester derivatives as anticancer against P-glycoprotein. Int J Pharm Pharm Sci 2014;7(2):485-8.

6. Nerdy, Putra ED, Haro G, Harahap U. In silico screening of hesperetin and naringenin ester derivatives as anticancer against phosphoinositide 3-kinase. Int J Pharmtech Res 2016;9(6):388-94.

7. Karsono, Patilaya P, Azisah N, Nerdy. Comparison of antimicrobial activity of red betel (Piper crocatum Ruiz and Pav) leaves nanoparticle and powder ethanolic extract against methicillin resistant Staphylococcus aureus. Int J Pharmtech Res 2015;8(4):696-701.

8. Masfria, Haro G, Nerdy, Mierza V, Wahyuni HS, Permata YM. Antimicrobials activity, antioxidants activity and analysis of active extract chemical compounds content of Moringa (Moringa oleifera Lam.) leaf. Int J Pharmtech Res 2016;9(11):110-20.

9. Nazliniwaty, Karsono, Zebua NF, Nerdy. Ethanol extract grapefruit peel (Citrus maxima Murr.) gel formulations with gelling agent durian seed 
gum and carboxy methyl cellulose. Pharm Chem 2016;8(20):207-13.

10. Nazliniwaty, Karsono, Zebua NF, Nerdy. Antioxidant activity and antiaging gel formulation grapefruit peel (Citrus maxima Merr.) ethanolic extract. Pharm Lett 2016;8(20):84-94.

11. Okereke CN, Iroka FC, Chukwuma MO. Phytochemical analysis and medicinal uses of Hibiscus sabdariffa. Int J Herb Med 2015;2(6):16-9.

12. Müller BM, Franz G. Chemical structure and biological activity of polysaccharides from Hibiscus sabdariffa. Planta Med 1992;58:60-7.

13. Ferreira JF, Luthria DL, Sasaki T, Heyerick A. Flavonoids from Artemisia апnи $\mathrm{L}$. as antioxidants and their potential synergism with artemisinin against malaria and cancer. Molecules 2010;15(1):3135-70.

14. Gulati M, Narula A, Vishnu R, Katyal G, Negi A, Ajaz I, et al. Plasmepsin II as a potential drug target for resistant malaria. Delhi Univ J Undergr Res Innov 2015;1(3):85-95.

15. Yuan L, Ziegler R, Hamann A. Inhibition of phosphoenolpyruvate carboxykinase gene expression by metformin in cultured hepatocytes. Chin Med J (Engl) 2002;115:1843-8

16. Ismail HM, Barton V, Phanchana $\mathrm{M}$, Charoensutthivarakul S,
Wong $\mathrm{MH}$, Hemingway $\mathrm{J}$, et al. Artemisinin activity-based probes identify multiple molecular targets within the asexual stage of the malaria parasites Plasmodium falciparum 3D7. Proc Natl Acad Sci U S A 2016;113:2080-5.

17. Terstappen GC, Reggiani A. In silico research in drug discovery. Trends Pharmacol Sci 2001;22:23-6.

18. Tegar M, Purnomo H. Tea leaves extracted as antimalaria based on molecular docking plants. Proc Environ Sci 2017;17(2013):188-94

19. Saptarini NM, Sitorus EY, Levita J. Structure-based in silico study of 6-gingerol, 6-ghogaol, and 6-paradol, active compounds of ginger (Zingiber officinale) as COX-2 inhibitors. Int J Chem 2000;5(3):12-8.

20. Bharath EN, Manjula SN, Vijaychand A. In silico drug design tool for overcoming the innovation deficit in the drug discovery process. Int $\mathrm{J}$ Pharm Pharm Sci 2011;3(2):8-12

21. Verbanac D, Jelic D, Stepanic V, Tatic I, Ziher D, Kostrun S. Combined in silico and in vitro approach to drug screening. Croatica Chem Acta 2005; 78(2):133-9. 